\title{
Tendencias en la modelización de cadenas de suministro: El enfoque referencial
}

\author{
María Victoria Labajo González \\ Universidad Pontificia Comillas-Icade \\ Martha Helena Carrillo Ramírez \\ Pontificia Universidad Javeriana \\ Ingeniería Industrial n. ${ }^{\circ}$ 29, 2011, ISSN 1025-9929, pp. 73-98 \\ Recibido: 8 de marzo del 2011 / Aprobado: 18 de marzo del 2011
}

\begin{abstract}
RESUMEN: La modelización es usada para el análisis y la comprensión de los sistemas, entre ellos, las cadenas de suministro. Específicamente, la modelización referencial facilita la generación de aproximaciones conceptuales a los elementos que componen una cadena, sus características y su dinámica de comportamiento $\mathrm{y}$, por lo tanto, se constituye en la base para el desarrollo de otros modelos más específicos de simulación o de programación matemática. El objetivo de este artículo consiste en ofrecer un panorama sobre la situación actual y las tendencias que se presentan en este campo de estudio, de modo que sirvan de referente para las investigaciones que se aborden en el futuro.
\end{abstract}

Palabras clave: cadena de suministro / modelización referencial / tendencias / simulación matemática / logística empresarial

\section{Referencial modeling for supply chains: future trends}

Aвstract: Modeling is used to understand systems, like supply chains. Particularly, referential modeling allows to create new conceptual bases about the elements of the supply chain and their dynamical behavior and consequently it is the base for developing more specific simulation models and mathematical programming. The purpose of this paper is to bridge a gap offering new perspectives, trends of study and challenges in supply chain modeling for future years. This paper contributes to provide to the scientific community a general overview of trends to lead upcoming research.

Keywords: supply chain / referential modeling / trends / mathematical simulation / business logistics 


\section{INTRODUCCIÓN}

Manejar la gran complejidad de un sistema empresarial es una tarea difícil debido al número de entidades involucradas, a la multiplicidad de sus relaciones y a la variedad de procesos. Esto, sumado a que las empresas se encuentran en permanente adaptación al entorno competitivo, da como resultado un ambiente con grandes retos para la administración actual y es entonces cuando la transformación de los procesos en modelos cobra gran relevancia, pues tales modelizaciones permiten contar con representaciones de la realidad que facilitan el análisis y el rediseño (Rocha dos Santos 2006: 181-182).

En el caso de las cadenas de suministro, el proceso de modelización es esencial para lograr su comprensión al facilitar la generación de aproximaciones conceptuales a sus elementos, sus características y su dinámica de comportamiento. Dentro de todas las posibilidades de modelos, los de tipo referencial se constituyen en la base de los demás, pues permiten establecer un marco general a partir del cual es posible particularizar. Constituyen los elementos para metodologías de administración, modelos de simulación, sistemas de información y modelos cuantitativos (Stefanovic y Stefanovic 2008: 485-503). Esta utilidad ha sido analizada y planteada por numerosos autores, entre ellos Min y Zhou (2002) y Van der Zee y Van der Vorst (2005).

Los modelos conceptuales o referenciales se pueden definir como herramientas que se construyen mediante prototipos del sistema en estudio y que proponen guías de análisis de los interrogantes planteados (Hernández et al. 2008). Se concretan generalmente en forma gráfica y se emplean para representar tanto la fenomenología estática, y las partes y sus propiedades, como la dinámica, los eventos y los procesos (Wand y Weber 2002). Su aporte para el estudio de cadenas de suministro ha sido determinante.

El nacimiento y la construcción de cada una de las técnicas de modelización se han realizado como parte de la búsqueda de respuestas a las problemáticas que se han presentado en las cadenas empresariales en cada momento de la historia. Los modelos referenciales son el reflejo de las necesidades de investigación en las cadenas de suministro.

Dada su importancia, es clave para los investigadores en el área de las cadenas de suministro conocer el panorama general del estado de la cuestión en el momento actual y un análisis de las principales 
tendencias en cuanto a los retos que se presentarán los próximos años y que implicarán nuevas búsquedas desde el punto de vista de la modelización referencial.

\section{CONSIDERACIONES METODOLÓGICAS}

Teniendo en cuenta la importancia de la modelización referencial en las cadenas de suministro, se decidió realizar una investigación cuyo objetivo general consistiría en analizar el estado actual de este tema y lograr un panorama sobre las posibles tendencias en su estudio para los años futuros.

La metodología de la investigación estuvo determinada por cuatro actividades consecutivas: estudio bibliográfico, análisis deductivo, estudio Delphi y análisis de tendencias.

La revisión bibliográfica se centró en artículos de revistas indexadas correspondientes a los meses transcurridos entre julio del 2008 a noviembre del 2010, referentes al tema de modelización referencial en cadenas de suministro. Una vez recolectados, se procedió a su análisis con dos fines: el primero de ellos, realizar el planteamiento de un resumen de la modelización referencial en la actualidad, que finalmente se construyó con las referencias que aparecen en el correspondiente capítulo, por ser las que brindaban un aporte más integral. El segundo, obtener un panorama sobre los temas que podrían considerarse como tendencias de estudio para los próximos años. Con este fin, los artículos se agruparon según las diferentes temáticas que presentaban, observándose una tendencia hacia el interés en ciertos temas en particular. Contando con esta información, y adicionalmente usando como herramienta para el ejercicio de prospectiva el método lógico deductivo por parte de conocedores del área, que permite llegar al planteamiento de hipótesis particulares a partir de la observación general, se realizó un primer acercamiento a cinco probables temas que posteriormente serían puestos a disposición de un grupo de expertos mediante un estudio tipo Delphi, con el fin de ser evaluados para su consideración final en el estudio de tendencias. Se incluyeron cuatro temas claramente identificados en los grupos de interés y uno adicional que, aunque contaba con presencia en la bibliografía, no era tan determinante, pero se decidió incluirlo por considerársele como una posibilidad a largo plazo. Los cuatro primeros fueron: estudios de modelización referencial en complejidad, estudios de modelización referencial en agilidad, estudios en 
cadenas colaborativas y cadenas virtuales, y estudios sobre la taxonomía u organización de los métodos de modelización referencial. La participación de cada uno de estos temas en los análisis posteriores sobre tendencias estaría determinada por los resultados del estudio Delphi.

Para el desarrollo del estudio tipo Delphi se contó con el apoyo de 12 expertos en el área de Logística cuyos perfiles aparecen en la tabla 1 y corresponden principalmente a personas con estudios muy superiores en Logística (maestría y doctorado) en un $92 \%$ de los casos y una persona ( $8 \%$ ) con licenciatura, ${ }^{1}$ aunque experto profesional en el tema.

Tabla 1

Perfiles de panelistas en el estudio Delphi

\begin{tabular}{ccccc}
\hline Número & Ubicación & Nivel máximo de estudios & Género & Área de desempeño \\
\hline 1 & Colombia & Maestría & Femenino & Educación \\
2 & Colombia & Maestría & Masculino & Educación \\
3 & España & Doctorado & Masculino & Educación \\
4 & España & Licenciatura & Masculino & Distribución \\
5 & España & Maestría & Femenino & Fabricación \\
6 & España & Maestría & Masculino & Servicios \\
7 & España & Maestría & Masculino & Distribución \\
8 & España & Maestría & Masculino & Independiente \\
9 & España & Maestría & Masculino & Fabricación \\
10 & España & Maestría & Femenino & Independiente \\
11 & España & Maestría & Masculino & Servicios \\
12 & España & Maestría & Masculino & Tecnología \\
\hline
\end{tabular}

Elaboración propia.

Se construyeron cinco afirmaciones, una por cada posible tendencia propuesta, que una vez probadas fueron utilizadas para elaborar una encuesta virtual que permitió el envío de los cuestionarios a los panelistas con el fin de que calificaran su nivel de acuerdo o desacuerdo con las afirmaciones. Esto se logró mediante una escala de valoración tipo Likert, en tres rondas sucesivas, durante las cuales fue posible que los expertos conocieran las opiniones del resto del grupo (de manera anónima) y pudieran modificar sus respuestas, si lo consideraban oportuno.

1 El estudio se realizó entre los meses de octubre del 2010 y enero del 2011. 
Las afirmaciones fueron las siguientes:

- A1. La modelización de cadenas de alta complejidad es una tendencia para los próximos años.

- A2. La modelización de cadenas cada vez más ágiles (flexibles y rápidas) es una tendencia para los próximos años.

- A3. La modelización de cadenas colaborativas es una tendencia para los próximos años.

- A4. La modelización de cadenas virtuales es una tendencia para los próximos años.

- A5. La organización sistemática de las múltiples propuestas de modelización actuales y futuras es una tendencia para los próximos años.

Adicionalmente, el cuestionario permitía la recolección de las opiniones de los panelistas, en cuanto a su acuerdo o desacuerdo con cada afirmación. El consolidado de estas opiniones durante las tres rondas, aparece a continuación y como se puede observar solo se presentó una opinión de desacuerdo, específicamente para la afirmación A3. Los demás aportes son acuerdos, pues apoyan de manera directa las afirmaciones propuestas.

Tabla 2

Resumen de opiniones de los panelistas

\begin{tabular}{lll}
\hline Pregunta & Aportes de los panelistas \\
\hline \multirow{2}{*}{ A1 } & Acuerdo & $\begin{array}{l}\text { La creciente competitividad requiere un entendimiento } \\
\text { mejor del funcionamiento de las cadenas complejas. }\end{array}$ \\
\cline { 2 - 3 } & Desacuerdo & No hay observaciones. \\
\hline A2 & Acuerdo & $\begin{array}{l}\text { La agilidad debe ser el factor diferenciador para que las } \\
\text { empresas compitan. }\end{array}$ \\
& & $\begin{array}{l}\text { La flexibilidad genera armonía con los diferentes es- } \\
\text { quemas de los procesos y adaptabilidad. La rapidez es } \\
\text { esencial, no se pueden esperar soluciones lentas y se } \\
\text { deben acondicionar los modelos de inmediato a medida } \\
\end{array}$ \\
& que las variables se modifican.
\end{tabular}

(continúa) 
(continuación)

\begin{tabular}{|c|c|c|}
\hline Pregunta & & Aportes de los panelistas \\
\hline \multirow[t]{2}{*}{ A2 } & Acuerdo & $\begin{array}{l}\text { - La tendencia es a flujo tenso, lo que hace que todo } \\
\text { tenga que ser más flexible y ágil. } \\
\text { - Los cambios y requerimientos del mundo global im- } \\
\text { plican flexibilidad para atender de manera eficiente } \\
\text { estas dinámicas en el entorno. } \\
\text { - En esa dirección se quiere avanzar pero la visibilidad } \\
\text { al cliente o al proveedor no está aceptada. }\end{array}$ \\
\hline & Desacuerdo & No hay observaciones. \\
\hline \multirow[t]{2}{*}{ A3 } & Acuerdo & $\begin{array}{l}\text { Win-Win, una colaboración fluida entre los integrantes } \\
\text { de la cadena hace que nuestras empresas trabajen } \\
\text { de una forma más hábil y eficiente, permitiendo mu- } \\
\text { chas más sinergias, ahorros de costes y mejoras en } \\
\text { la calidad. }\end{array}$ \\
\hline & Desacuerdo & La colaboración es un requisito en el futuro de la SC. \\
\hline \multirow[t]{2}{*}{ A4 } & Acuerdo & $\begin{array}{l}\text { Sí es una tendencia debido a que el software y los } \\
\text { sistemas de modelación son cada vez más versátiles } \\
\text { y fáciles de utilizar. Permiten observar comporta- } \\
\text { mientos que son difíciles de medir de otra manera. }\end{array}$ \\
\hline & Desacuerdo & No hay observaciones. \\
\hline \multirow[t]{2}{*}{ A5 } & Acuerdo & $\begin{array}{l}\text { No es suficiente la recoleccion de informacion, es } \\
\text { necesaria la organización clara y sistemática para la } \\
\text { oportuna toma de decisiones. }\end{array}$ \\
\hline & Desacuerdo & No hay observaciones. \\
\hline
\end{tabular}

Elaboración propia.

Para el análisis de los resultados numéricos obtenidos en cada una de las tres rondas de discusiones realizadas, se utilizaron los indicadores cuartil primero Q1, cuartil segundo Q2 o mediana, cuartil tercero Q3 y rango. El primer cuartil es el valor en el cual o por debajo del cual queda un cuarto (25\%) de todos los valores de la sucesión ordenada; el tercer cuartil es el valor en el cual o por debajo del cual quedan las tres cuartas partes $(75 \%)$ de los datos. El segundo cuartil corresponde a la mediana y el rango es una medida de la dispersión de las respuestas y se calcula mediante la resta simple del último valor menos el primero. 
Con el fin de facilitar el análisis de los datos y contar con un método de referencia, se plantearon las siguientes categorías de resultados: Sobresaliente: Mediana (4-5), Rango (1-2); Suficiente: Mediana y Rango (3); Inferior: Mediana (1-2), Rango (4-5).

Tabla 3

Resultados de estudio Delphi

\begin{tabular}{|l|c|c|c|c|c|c|c|c|c|c|c|c|}
\hline & \multicolumn{4}{|c|}{ Ronda 1 } & \multicolumn{4}{c|}{ Ronda 2 } & \multicolumn{4}{c|}{ Ronda 3 } \\
\hline & Q1 & Med & Q3 & Rango & Q1 & Med & Q3 & Rango & Q1 & Med & Q3 & Rango \\
\hline A1 & 2 & $\mathbf{3}$ & 3,25 & 3 & 2 & $\mathbf{3}$ & 4 & 2 & 2,5 & $\mathbf{3}$ & 4 & 3 \\
\hline A2 & 4 & $\mathbf{4}$ & 5 & 3 & 4 & $\mathbf{4}$ & 5 & 2 & 4 & $\mathbf{4}$ & 5 & 2 \\
\hline A3 & 2,75 & $\mathbf{3 , 5}$ & 4 & 3 & 3 & $\mathbf{4}$ & 4 & 2 & 3,5 & $\mathbf{4}$ & 4 & 2 \\
\hline A4 & 3 & $\mathbf{4}$ & 4 & 3 & 3 & $\mathbf{4}$ & 4 & 2 & 3 & $\mathbf{4}$ & 4 & 2 \\
\hline A5 & $\mathbf{2}$ & $\mathbf{3}$ & 3,5 & 3 & 3 & $\mathbf{3}$ & 4 & 2 & 3 & $\mathbf{3}$ & 4 & 3 \\
\hline
\end{tabular}

Elaboración propia.

En cuanto a la primera ronda, se observa que todos los rangos estuvieron en la categoría de suficiente, lo mismo que tres de las medianas. Las otras dos medianas clasificaron en el grupo de sobresaliente. No se presentó ninguno en nivel inferior.

En lo que respecta a la segunda ronda, todos los rangos estuvieron en la categoría sobresaliente, lo mismo que tres de las medianas. Las otras dos medianas clasificaron como suficiente. No se presentó ningún nivel inferior. El cuadro también permite observar cómo la mediana permaneció constante en todas las afirmaciones a excepción de una en la cual hubo un incremento en la tercera con respecto a la primera ronda.

En lo que se refiere al rango, en la última ronda las cinco afirmaciones presentan un rango final menor o igual al presentado en la primera ronda, lo que indica cómo, en el transcurso del proceso, los panelistas fueron unificando sus ideas. Las preguntas A1 y A5 presentaron variación en el rango durante la segunda ronda.

Un análisis específico de la tercera y última ronda permitió obtener los siguientes resultados: 
Figura 1

Resultados de la ronda tres

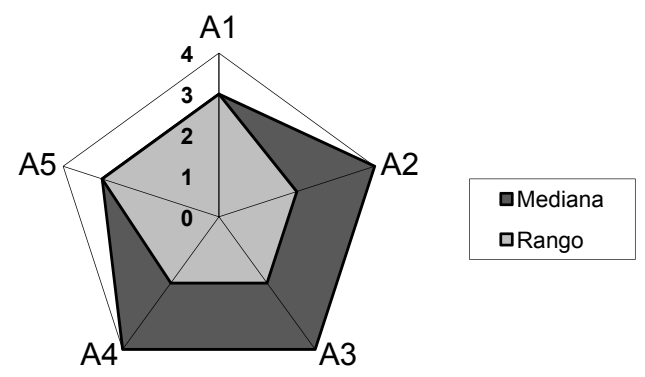

Elaboración propia.

En relación tanto con la mediana como con el rango se obtuvieron tres resultados sobresalientes correspondientes a las afirmaciones A2, A3 y A4, dos suficientes correspondientes a A1, A5 y ninguno de tipo inferior.

Ante este resultado, se concluyó que no se debería descartar ninguna de las afirmaciones propuestas en el estudio Delphi, y por lo tanto las cinco fueron incluidas en el análisis como posibles tendencias en el desarrollo de la modelización referencial de las cadenas de suministro. Posteriormente se procedió a un examen un poco más profundo de su significado en el panorama actual, a partir del cual se realizó el resumen presentado a continuación.

\section{PANORAMA ACTUAL DE LA MODELIZACIÓN REFERENCIAL}

Durante las décadas de 1970 y 1980 se produjo el gran despertar en la modelización referencial, principalmente estimulado por el desarrollo de sistemas de bases de datos y técnicas de análisis de sistemas. Aunque durante la década de 1990 disminuyó un poco el énfasis en el tema, en la actualidad muestra un renovado interés (Wand y Weber 2002: 363-376).

Cada época ha presentado sus propias versiones de modelos referenciales para analizar procesos según las necesidades del momento. A continuación se presenta un resumen con algunas de las propuestas que caracterizaron cada década: 
Tabla 4

Síntesis del desarrollo y evolución de la modelización

\begin{tabular}{ll}
\hline \multicolumn{1}{c}{ Década } & \multicolumn{1}{c}{ Modelos } \\
\hline 1970 & DFD y las redes Petri \\
1980 & Métodos $\mathrm{CIM}^{2}$ \\
A fines de 1980 & BPR orientado a procesos, Cimosa \\
1990 & $\mathrm{ERP}^{3}$, workflow, orientación a objetos (UML), ontologías IDEF5 \\
\hline
\end{tabular}

Fuente: Rocha dos Santos et al. (2006).

Aguilar-Saven (2004) ofrece una breve definición de los más significativos:

- DFD (Data Flow Diagrams [Diagrama de Flujo de Dastos]) son diagramas que muestran el flujo de información de un sitio a otros y describen los procesos mostrando cómo se relacionan entre sí por medio de almacenes de datos.

- Redes Petri (PN por su locución anglosajona) es un lenguaje orientado a gráficas para diseño, especificación, simulación y verificación de sistemas. Es particularmente usado cuando se trata de un grupo de procesos que se debe sincronizar y comunicar.

- La arquitectura de sistema abierto para control integrado de manufactura (CIM) es una metodología de apoyo para la integración de la empresa, basada en sistemas de cómputo. Un ejemplo es Cimosa.

- La reingeniería de procesos del negocio (BPR por su locución anglosajona) busca ofrecer una herramienta para el diseño de procesos.

- La metodología ARIS corresponde a un tipo de DFD que ha sido principalmente usado para el desarrollo e implantación de los sistemas Enterprise Resource Planning (ERP) (Shen et al. 2004: 307323).

- Las técnicas de workflow generalmente son definidas como la automatización computarizada de procesos del negocio, durante la cual los documentos, la información o las tareas son transmitidas de un participante a otro de acuerdo con un conjunto de reglas procedi-

2 Uno de los más conocidos es el denominado GRAI.

3 ARIS es un ejemplo. 
mentales. Consiste en un flujo de tareas entre las aplicaciones del computador y las personas en la organización.

- Los métodos orientados a objetos son una herramienta para modelizar y programar procesos descritos como objetos, los cuales son transformados por actividades a lo largo del proceso. La principal entidad son los objetos, cada uno de los cuales posee atributos y operaciones.

Todas estas aportaciones integran el panorama actual de la modelización descriptiva, pues se conjugan de diferentes formas para dar vida a modelos más complejos o, en sus presentaciones más simples, son usadas en las empresas como herramientas de trabajo diario.

La constante aparición de nuevos modelos justifica los intentos de abordar su clasificación. Algunas propuestas presentan un panorama muy general, como en el caso de Salvendy (2001), quien sostiene que los acercamientos teóricos al tema pueden considerarse de dos clases:

a) Orientados a procesos.- Este tipo de modelización determina en primera instancia las actividades que se ejecutan para cumplir los objetivos y las entidades que toman parte de dicha ejecución.

b) Orientados a objetos.- Este tipo de modelización establece en primera instancia las entidades que conforman el sistema, las que luego son descritas a través de objetos.

Otros autores, como Camarinha-Matos y Afsarmanesh (2007), proponen una taxonomía muy completa de los diferentes acercamientos teóricos y conceptuales existentes en la actualidad sobre modelizaciones de sistemas complejos como las redes de suministro.

Considerando la forma mediante la que se desee analizar el sistema, sus elementos y sus relaciones, se plantea una subdivisión general según cuatro dimensiones: las relativas a los componentes, a los comportamientos, las estructurales y las funcionales.

El resultado de esta propuesta es una detallada red que va desde los enfoques más generales en el centro, al detalle de enfoques particulares en los extremos, en los que encontramos, por ejemplo, la teoría de juegos y las redes Petri como parte de las alternativas. 


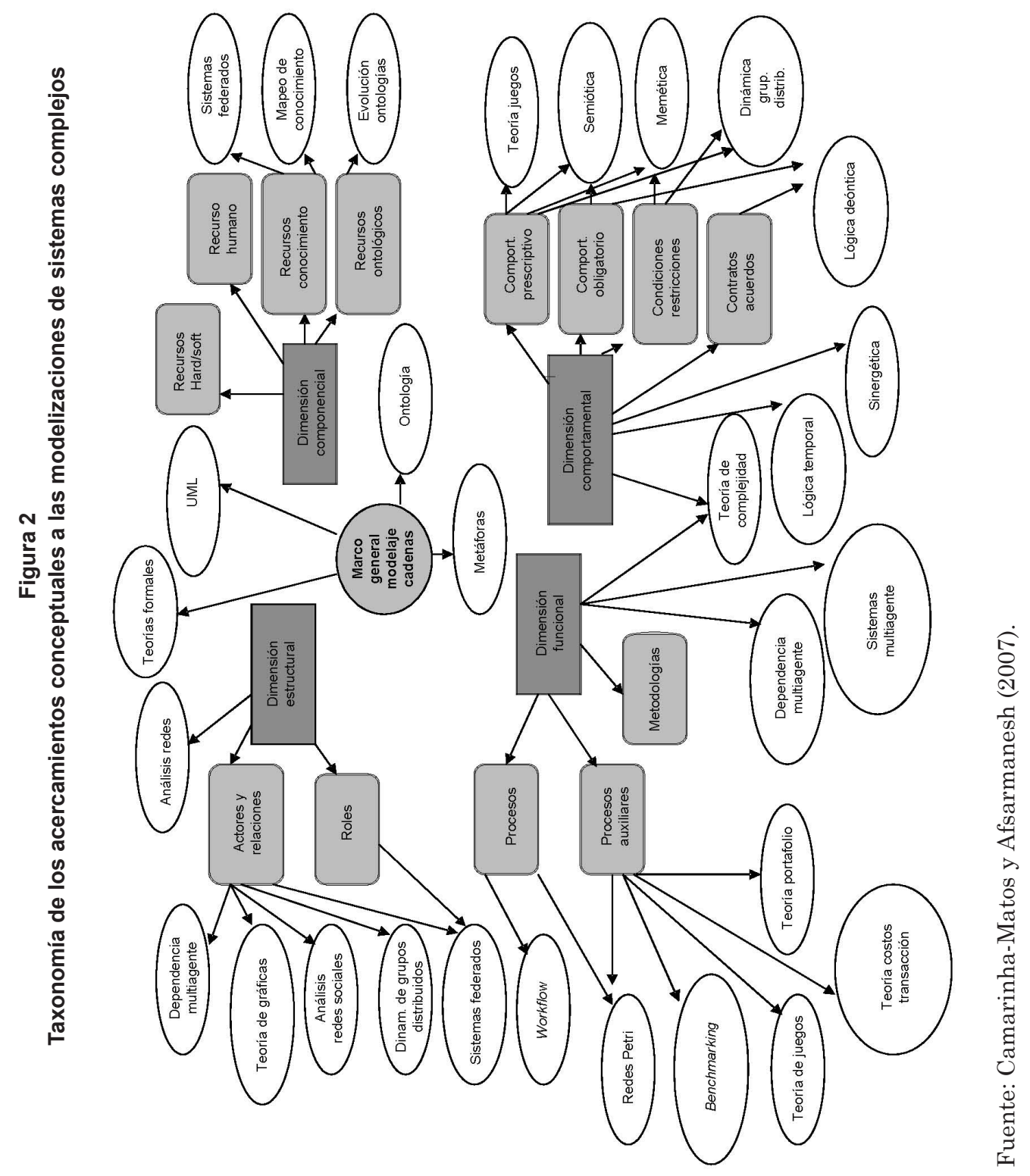


Un ejemplo práctico de modelización de tipo referencial, muy utilizado en la actualidad, es el SCOR (Supply Chain Operations Reference Model). Este modelo utiliza diferentes submodelos referenciales de acuerdo con el propósito que busca, entre ellos se encuentran (Supply Chain Council 2010: 21):

- Diagramas de alcance del proyecto.

- Mapas geográficos de flujo de material.

- Diagrama de hilos para flujo de material, nivel 2.

- Workflow o modelo de proceso, nivel 3.

A continuación se presenta un ejemplo de modelo de proceso:

Figura 3

Modelo de proceso (SCOR)

rutas de envío

pedidos

De fuente o producción

\section{D1.2 Recibir ingresar $y$ validar pedido}

D1.3 Reservar fecha

D1.12 Despachar producto

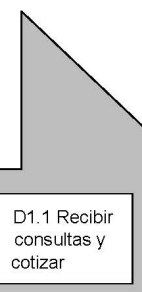

D2. Entrega producto contra orden

D1.13 Recepción por el cliente $y$ verificación

Fuente: Supply Chain Council (2010). 


\section{TENDENCIAS EN LA MODELIZACIÓN REFERENCIAL DE CADENAS DE SUMINISTRO}

A continuación se exponen los posibles retos en modelización referencial de cadenas de suministro que se presentarán en los próximos años y que serán incentivos para la generación de nuevas investigaciones. Los tres primeros obtuvieron una mediana y un rango "sobresaliente" en el estudio Delphi, lo que parece indicar que los expertos logran ver claramente la tendencia para nuevos estudios, desde el momento actual. Los dos siguientes corresponden a temas que recibieron resultados "suficientes" por parte de los expertos, lo que indica que aunque los ven como tendencia, todavía está por construirse un panorama más claro del tema en el futuro.

\subsection{Modelización en agilidad}

La agilidad es una de las características fundamentales de las cadenas de suministro para sobrevivir en un mercado en constante cambio, propio de esta época donde los ciclos de vida del producto son cada vez más cortos y fuerzas externas como las medioambientales aumentan la incertidumbre y, por lo tanto, el riesgo. En la era de la competencia basada en el tiempo [time based], las cadenas de suministro deben tener la habilidad de responder a la demanda de los clientes con mayor agilidad y sincronizar el suministro durante los picos de esta.

Cultivar la agilidad permite a la empresa y a sus socios responder rápidamente a los cambios del mercado y a potenciales trastornos en la cadena de suministro (Braunsheidel y Suresh 2008: 22).

Swafford et al. (2000) proponen un modelo para describir la agilidad, en el cual se plantean como variables determinantes las siguientes: flexibilidad en el desarrollo de productos, flexibilidad en los recursos, flexibilidad en la fabricación y flexibilidad logística, aludiendo asimismo a la flexibilidad en las tecnologías de información y a la influencia del entorno competitivo global.

Según Agarwal et al. (2007), las variables para asegurar la agilidad son: sensibilidad al mercado, velocidad de entrega, exactitud de datos, introducción de nuevos productos, planificación centralizada y colaborativa, integración de procesos, uso de herramientas TIC, reducción de tiempos de entrega [lead times], mejora del nivel de servicio, minimización de costes, satisfacción de consumidor, mejora de la calidad, mini- 
mización de incertidumbre, desarrollo de la confianza y minimización de la resistencia al cambio.

Una de las pocas metodologías diseñadas para lograr un modelo de empresa ágil se denomina ISM [Interpretive Structural Modeling] y consiste en un proceso de aprendizaje interactivo mediante el cual una serie de elementos se constituyen dentro de un modelo sistémico que representa la estructura de un problema complejo mediante gráficos y palabras (Agarwal et al. 2007: 443-457).

Como ejemplo aplicado al sector empresarial, se encuentra el artículo de Yang y Li (2002) sobre la valoración de la agilidad en una empresa manufacturera. En este análisis se realizó la valoración mediante un sistema indexado conformado por tres aspectos: administración de la organización, diseño del producto y procesamiento, como propuesta de indicadores para medir la agilidad y por lo tanto para ser considerados en el momento de diseñar nuevos modelos.

A pesar de estos aportes, este tema se encuentra todavía en fase de investigación, pues solo en los últimos años la necesidad de agilidad se ha revelado como imperiosa para sobrevivir en el mercado. Aunque existen algunos estudios al respecto, también son claras las carencias. Por ejemplo, la literatura actual de cadenas de suministro describe la dependencia de la agilidad respecto al desempeño de otras variables; sin embargo, la interrelación entre todo el conjunto de variables ha sido tomada muy poco en cuenta y, por lo tanto, ofrece un campo de investigación para la construcción de nuevos modelos que las integren (Agarwal et al. 2007: 443-457).

Ante este panorama, es posible concluir que nuevas investigaciones son necesarias para realizar aportaciones complementarias al tema, que está aún en sus etapas iniciales de estudio.

\subsection{Modelización en redes colaborativas}

Redes colaborativas [Collaborative Networks Organizations] son entidades complejas, cuyo correcto entendimiento, diseño, implantación y manejo requieren la integración de diferentes perspectivas de modelización.

El objetivo de una red colaborativa es obtener ventajas competitivas mediante la mejora de todo el desempeño general, considerando una perspectiva holística de las cadenas de suministro. Esto se logra me- 
diante la alineación de todas las actividades desde planificación hasta operación en función de los objetivos de la red (Angerhofer y Angelides 2006: 283-301).

Según Kim (2006), las principales características que debe tener una red de este tipo y que aparecen en la tabla son las siguientes:

Tabla 5

Atributos de una red colaborativa

\begin{tabular}{ll}
\hline Atributos & Características \\
\hline Estructura & Red, no lineal, flexible, buena respuesta \\
Personal & Colaborativa, confiable \\
Alianzas & win-win \\
Recursos & Outsourcing \\
Contabilidad & Basada en stakeholders \\
Procesos & Integrados, sincronizados \\
Tecnología & Conectada, compatible \\
Satisfacción & Personalización \\
Conocimiento & Toda la cadena, explícito y tácito \\
Medida & Objetivos comunes \\
Resultados & Optimización global \\
\hline
\end{tabular}

Fuente: Kim (2006).

Si se revisa al detalle cada uno de estos elementos, es posible observar que abarcan una amplia gama de temas relacionados con diversas ciencias y que su desarrollo implica el establecimiento de una serie de estrategias complejas que serían la base a partir de la cual es posible realizar modelizaciones de nuevas cadenas que deseen implementar procesos colaborativos.

Ke y Wei (2007) plantean que compartir información es uno de los elementos más importantes. Las investigaciones al respecto han encontrado que los acuerdos de colaboración exitosos se han caracterizado por diseños adecuados de comunicaciones, mediante tecnologías de la información (Wareham et al. 2005: 201-227), con lo cual se concluye que futuros estudios en este campo supondrían una importante aportación para las redes de suministro.

Por su parte, Quinn (2000) plantea en su artículo las razones, los facilitadores y los impedimentos para la colaboración entre los que se 
destaca la natural resistencia al cambio y la desconfianza en el poder de los socios (Ke y Wei 2007: 297-308).

Como ejemplo de este tema aplicado a la industria es posible referirse al caso de Bahinipati et al. (2009), quienes presentan en su artículo conclusiones sobre una empresa de semiconductores en la cual se realizan esfuerzos dirigidos a consolidar sus esquemas colaborativos mediante la elaboración de nuevos modelos con el fin de evaluar el grado de colaboración, específicos para este tipo de empresas.

En resumen, el panorama de aportes sobre el tema de modelización en colaboración permite concluir que aunque este es un tema relativamente nuevo, un gran número de herramientas y teorías han sido desarrolladas por diversas disciplinas y tienen un gran potencial en este campo (Camarinha-Matos y Afsarmanesh 2007: 529-542), lo que permitiría nuevos desarrollos acordes con las necesidades de cada empresa.

\subsection{Modelización en cadenas virtuales [e-chain]}

Desafortunadamente, las redes de suministro sufren con frecuencia de una información incompleta e inexacta y, en el peor de los casos, la información no está disponible dónde y cuándo se necesita. Tal entropía crea errores y limita la respuesta de procesos en los que se puede presentar por ejemplo que hay demasiado o muy poco inventario. El e-business puede cambiar la definición actual de los modelos de las cadenas de suministro y mejorar estos problemas (Wareham et al. 2005: 201-227).

Las principales iniciativas actuales de e-business para cadenas de suministro se resumen en la tabla que proponen Kim y Park (2008) (tabla 6).

Según lo plantean estos autores, algunos estudios han demostrado que es difícil encontrar ejemplos de empresas con desarrollo de modelos de e-business que hayan penetrado más allá de un nivel superficial. De hecho, se pueden definir tres niveles de penetración basados en el grado de madurez en la organización: e-commerce, e-business y $e$-chain, los tres están presentes actualmente, lo que significa retos en materia de investigación. En la etapa de e-commerce, los proveedores establecen escaparates virtuales [storefronts], a través de los cuales los proveedores buscan información de productos y servicios y realizan 
Tabla 6

Iniciativas de e-business

\begin{tabular}{lll}
\hline $\begin{array}{l}\text { E-business } \\
\text { iniciativas }\end{array}$ & El efecto de e-business \\
\hline E-technology & EDI & $\begin{array}{l}\text { Diferentes tipos de EDI dependiendo del tipo } \\
\text { de tecnología TIC }\end{array}$ \\
E-documentation & $\begin{array}{l}\text { Proceso de creación y flujo del documento } \\
\text { Seguimiento de documentos con fines de } \\
\text { auditoría }\end{array}$ \\
& E-auditing & $\begin{array}{l}\text { Permite a un gran número de proveedores ofre- } \\
\text { cer sus productos }\end{array}$ \\
& EC-agent & $\begin{array}{l}\text { EC-agent: implementa comandos de selección y } \\
\text { contrata directamente con proveedores }\end{array}$ \\
& Pago online & Pago electrónico a proveedores \\
E- collaboration & Nivel de integración & $\begin{array}{l}\text { Más allá de EDI se requiere lograr una integra- } \\
\text { ción estratégica. ERP es una ayuda }\end{array}$ \\
& Entregas JIT & $\begin{array}{l}\text { Ya se ha realizado en parte la integración de JIT } \\
\text { en la filosofía general de integración de cadenas }\end{array}$
\end{tabular}

Fuente: Kim y Park (2008).

transacciones de forma automatizada. En e-business se busca colaboración y compartir información entre los socios de una cadena de suministro lineal y esto es soportado con sistemas electrónicos que incluyen internet. En e-chain una cadena de suministro lineal toma la forma de red, lo que facilita el crecimiento de los negocios $\mathrm{B} 2 \mathrm{~B}^{4}$ dado que existe una mejor información, que reduce los tiempos de espera y de transferencia.

Un ejemplo de la importancia de este tema para la industria es presentado por Wang y Chang (2010). En este estudio se exponen dos aplicaciones en el sector textil, que ejemplifican como las empresas pueden

4 Es la abreviatura comercial de la expresión anglosajona Business to Business y que significa comercio electrónico de empresa a empresa. 
hacer uso de la modelización de una organización virtual para integrar sus actividades.

En conclusión, aunque en la actualidad las cadenas virtuales no representan la generalidad, su forma de operar se manifiesta como una tendencia cada vez más consolidada. Existe entonces una oportunidad para la modelización referencial en este camino hacia la implantación de modelos e-chain.

\subsection{Taxonomía de los principales métodos de modelización}

Dada la gran variedad de acercamientos teóricos que han surgido en los últimos años para el tema de la modelización en cadenas de suministro, se presenta una oportunidad en su proceso de clasificación y estructuración. Al respecto, algunos autores como Aguilar-Saven (2004) subrayan que hay una considerable confusión en la terminología que se usa y en el sentido que se le otorga a cada denominación y nomenclatura. Es también palpable, como apunta el mencionado autor, la necesidad de contar con estudios comparativos que mediante el análisis de los diferentes modelos y sus características, construyan un marco referencial para que futuros investigadores cuenten con un panorama claro de las posibilidades, ventajas, desventajas, criterios, opciones, aplicabilidad, alcance y compatibilidad de cada uno de los modelos y de sus posibles integraciones con otros.

Existe también un campo de oportunidades relacionado con el avance y mejora de los conceptos de modelización en sí mismos, ya no de su aplicación directa, como lo resumen Wand y Weber (2002) en la siguiente tabla:

Tabla 7

Oportunidades de investigación asociadas a los principales conceptos de modelización

\begin{tabular}{ll}
\hline Elementos de investigación & Oportunidades de investigación \\
\hline Gramáticas de modelización referencial & $\begin{array}{l}\text { Hacer seguimiento a las fortalezas y debilidades } \\
\text { de nuevas y existentes gramáticas } \\
\text { Determinar cuál combinación de gramáticas da } \\
\text { mejor soporte a los usuarios }\end{array}$ \\
\hline Métodos de modelización referencial & $\begin{array}{l}\text { Evaluar el grado de adecuación de los métodos } \\
\text { para modelar }\end{array}$ \\
\hline
\end{tabular}

(continúa) 
(continuación)

\begin{tabular}{ll}
\hline Elementos de investigación & Oportunidades de investigación \\
\hline Códigos de modelización referencial & $\begin{array}{l}\text { Evaluar diferentes combinaciones de código } \\
\text { para determinar la que mejor se adapte a las } \\
\text { necesidades }\end{array}$ \\
\hline $\begin{array}{l}\text { Diferencias individuales en el contexto } \\
\text { de la modelización referencial }\end{array}$ & $\begin{array}{l}\text { Desarrollar herramientas de soporte a la mode- } \\
\text { lización conceptual }\end{array}$ \\
\hline Tareas & Evaluar modelos para diferentes tareas \\
\hline Agenda social & $\begin{array}{l}\text { Determinar los costes y beneficios de adoptar } \\
\text { determinado modelo }\end{array}$ \\
\hline
\end{tabular}

Fuente: Wand y Weber (2002).

Un ejemplo de la importancia del tema y su tendencia para continuar presente en las investigaciones de los próximos años aparece en el artículo publicado por Estampe et al. (2010), en el que se analizan diversos modelos usados actualmente mediante un marco de referencia conformado por siete niveles, el cual constituye un aporte para los gerentes como herramienta para determinar cuáles modelos se ajustan de manera más precisa a las necesidades reales de una determinada organización.

Es posible concluir que se requiere contar con permanentes estudios, clasificaciones y evaluaciones de los avances que se vayan realizando en torno a modelización referencial de cadenas de suministro, pues es un campo con amplio potencial por desarrollar y permite facilitar los procesos de investigación de la comunidad científica sobre el tema.

\subsection{Modelización en complejidad}

En los últimos años ha aumentado de manera acelerada la globalización de las empresas debido a que se ha descubierto que mediante esta práctica reducen costes, aumentan utilidades y mejoran el desempeño general. Sin embargo, también se ha notado que estas cadenas de suministro son más difíciles de manejar pues implican la coordinación de tiempos de entrega ${ }^{5}$ más largos, originados en los múltiples transpor-

5 Este concepto implica el periodo de tiempo comprendido entre la iniciación de un proceso y su finalización. Es particularmente usado en el entorno empresa- 
tes a lo largo y ancho del planeta. Por otro lado, la inclusión de diferentes lenguas y culturas, cada una de las cuales está definida por sus propias condiciones y variables, da como resultado una disminución general de la efectividad en toda la red; panorama que se complementa con la mala situación de la infraestructura de vías y comunicaciones en algunos países que no es la más idónea para asegurar una real efectividad en los flujos de las cadenas de suministro (Meixell y Gargeya 2005: 531-550). Esta problemática, vista en su totalidad, genera un aumento permanente en la complejidad de los sistemas logísticos.

En una cadena de suministro, la complejidad podría definirse como la suma de diversos factores, entre los que encontramos: una gran variedad de productos y de estados de los sistemas, un número elevado de elementos y subsistemas y un grado de conectividad e interacción entre esos elementos que la hace impredecible y de alta incertidumbre.

Según Wu et al. (2007), la complejidad operacional es una fuente mayor de costes y, por lo tanto, debe ser un punto focal a considerar en los procesos de modelización de operaciones.

Como caso aplicado de este tema se encuentra el presentado por $\mathrm{Li}$ et al. (2009), quienes en su estudio plantean un modelo cuantitativo de definición de capacidades en diferentes locaciones, para una cadena compleja, basado en el modelo referencial correspondiente.

En la actualidad, existen varios enfoques conceptuales que pueden ayudar al entendimiento y modelización de la complejidad. Uno de ellos es la comprensión de las cadenas como un sistema ampliado [system wide] (Chan y Chan 2005: 998-1006). Esto implica que, dado que la cadena tiene múltiples actores y actividades, la red resultante es un sistema complejo y debe buscar sinergias de modo que se logre su funcionamiento como un solo organismo.

Según los mismos autores, existen otros enfoques para facilitar el estudio de la complejidad y entre ellos encontramos la 'asignación de heterarquía'. En este caso no existe un subsistema único gobernante sino que, por el contrario, cada subsistema ejerce ciertas influencias sobre los demás, constituyendo sistemas débilmente acoplados.

rial referido a los tiempos que debe esperar un cliente desde que coloca el pedido hasta que recibe el producto finalizado. Su locución anglosajona es 'lead time', utilizada muy frecuentemente. 
Esto se debe a que se registra una interacción descentralizada impulsada por diferentes intereses particulares que incluso pueden ser contradictorios, lo que genera fenómenos con niveles semiindependientes uno del otro y entremezclados entre sí en formas asimétricas (Morales 2007).

Asimismo, los mencionados autores plantean el análisis de sistemas Multiagente ${ }^{6}$ (MAS, sigla de la locución anglosajona Multi Agent Chain [Cadena de suministro multiagente]) como otra opción para analizar los sistemas complejos. MAS es una derivación de la inteligencia artificial distribuida que se concentra en el desarrollo de modelos de organización estratégica para la solución de problemas y mecanismos de cooperación y coordinación, para un rango de módulos de solución de problemas basados en conocimiento.

Según lo explican Meixell y Gargeya (2005), a pesar de estos aportes, los acercamientos al tema de la modelización en complejidad aún son parciales, pues no logran captar la totalidad de una cadena de suministro global ni su gran variabilidad.

Todos los anteriores planteamientos y propuestas permiten ver que en este tema existe una dinámica en desarrollo, que se presenta como un gran campo de ideas para nuevas investigaciones.

\section{TABLA RESUMEN DE TENDENCIAS}

A continuación se presenta una tabla resumen de los principales autores en cada tendencia y los aportes que realizan para el desarrollo del tema:

6 Siglas correspondientes a la locución anglosajona Multi Agent Supply Chain que puede traducirse como Cadena de suministro multiagente. 


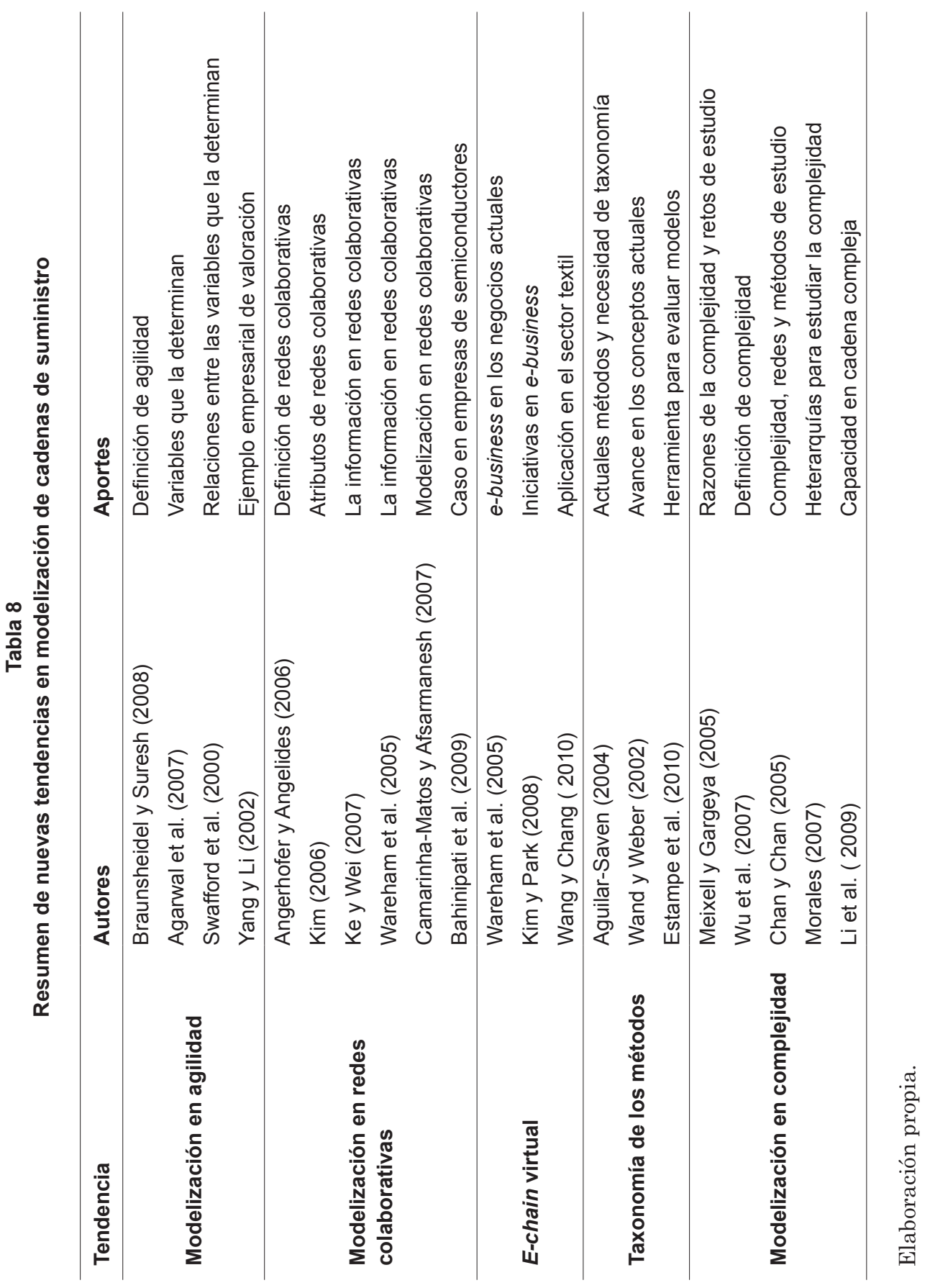




\section{CONCLUSIONES}

En los últimos años se han realizado importantes aportaciones al tema de modelización de cadenas de suministro desde diferentes disciplinas científicas y heterogéneos puntos de vista, lo que ha hecho que se amplíe el espectro de posibles soluciones a las problemáticas presentadas. Cada periodo histórico implica nuevos trabajos y estudios en relación con los modelos necesarios para cubrir las necesidades imperantes en los negocios del momento.

Por supuesto, en la época actual también existen algunos retos que presionan el desarrollo de nuevos estudios de modelización de cadenas de suministro. Algunos de ellos han empezado a desarrollarse y ya se encuentran referenciados en artículos científicos, aunque todavía falta mucho para su total cubrimiento. Otros, por el contrario, se encuentran lejos del total desarrollo y aún no es claro su futuro, pero de todas formas, todos ellos constituyen el panorama para los próximos años en cuanto a modelización referencial se refiere.

Según el estudio Delphi, en el primer grupo encontramos la modelización de cadenas ágiles, de cadenas colaborativas y de cadenas virtuales. En el segundo grupo se encuentran los temas de taxonomía de modelos y modelización en complejidad.

Conocer estas tendencias podrá servir de guía para nuevas profundizaciones e investigaciones en el tema de modelización referencial de cadenas de suministro en los años futuros.

\section{BIBLIOGRAFÍA}

Agarwal, A.; Shankar, R. y M. K. Tiwari (2007). "Modeling agility of supply chain”. Industrial Marketing Management, vol. 36.

Aguilar-Saven, R. (2004). "Business process modeling: review and framework". International Journal of Production Economics, vol. 90.

Angerhofer, B. y M. Angelides (2006). "A model and a performance measurement system for collaborative supply chains". Decision Support Systems, vol. 42.

Bahinipati, B.; Kanda, A. y S. Deshmukh (2009). "Horizontal collaboration in semiconductor manufacturing industry supply 
chain: An evaluation of collaboration intensity index". Computers \& Industrial Engineering, vol 57.

Braunsheidel, M. y N. Suresh (2008). "The organizational antecedents of a firm's supply chain agility for risk mitigation and response". Journal of Operations Management, vol. 27.

Camarinha-Matos, L. y H. Afsarmanesh (2007). "A comprehensive modeling framework for collaborative networked organizations". Journal of Intelligent Manufacturing, vol. 18.

Chan, F. y H. Chan (2005). "The future trend on system - Wide modeling in supply chain studies". International Journal of Advanced Manufacturing Technology, vol. 25.

Estampe, D.; Lamouri, S.; Paris, J. y S. Brahim-Djelloul (2010). "A framework for analysing supply chain performance evaluation models". International Journal of Production Economics, vol. 15.

Hernández, J.; Mula, J.; Ferriols, F. y R. Poler (2008). “A conceptual model for the production and transport planning process: An application to the automotive sector". Computers in Industry, núm. 59.

Ke, W. y K. Wei (2007). "Factors affecting trading partners knowledge sharing: using the lens of transaction cost economics and sociopolitical theories". Electronic Commerce Research and Applications, vol. 6 .

Kim, D. (2006). "Process chain: A new paradigm of collaborative commerce and synchronized supply chain”. Business Horizons, vol. 49 .

Kim, S. y S. Park (2008). "Development of a three - echelon SC model to optimize coordination cost". European Journal of Operational Research, vol. 184.

Li, H.; Hendry, L. y R. Teunter (2009). "A strategic capacity allocation model for a complex supply chain: Formulation and solution approach comparison". International Journal of Production Economics, vol. 121.

Meixell, M. y V. Gargeya (2005). "Global supply chain design: a literature review and critique". Transportation research, vol. 41. 
Min, H. y G. Zhou (2002). "Supply chain modeling: past, present and future”. Computers and Industrial Engineering, vol. 43.

Morales, F. (2007). "Los conceptos de jerarquía y heterarquía en el análisis del desarrollo local". México: Universidad Autónoma de México. <http://www.proimmse.unam.mx/doctos/Morales_ UAMI0406.pdf>. [Consulta: 15 de noviembre del 2010.]

Quinn, Francis (2001). "Collaboration most than just technology". Supply Chain Management Review.

Rocha dos Santos, L.; Vasconcelos, S. y R. De Campos (2006). "Research and practical issues of enterprise information systems". International Federation for Information Processing, vol. 205.

Salvendy, G. (2001). Handbook of industrial engineering technology and operation management. 3. ${ }^{a}$ edición. Nueva York: John Wiley \& Sons.

Shen, H.; Wall, B.; Zaremba, M.; Chen, Y. y J. Browne (2004). "Integration of business modeling methods for enterprise information system analysis and user requirements gathering". Computers in Industry, vol. 54.

Stefanovic, D. y N. Stefanovic (2008). "Methodology for modeling and analysis of supply chain". Journal of Intelligent Manufacturing, vol. 19 .

Supply Chain Council (2010). "SCOR 10.0". Overview Booklet. SCC.

Swafford, P.; Ghosh, S. y N. Murthy (2000). "A model of global supply chain agility". Georgia: Georgia Institute of Technology.

Van der Zee, D. y J. Van der Vorst (2005). "A modeling framework for supply chain simulation: opportunities for improved". Decision Sciences, vol. 36.

Wand, Y. y R. Weber (2002). "Research commentary: Information systems and conceptual modeling - a research". Information Systems Research, vol. 13.

Wang, W. y H. Chang (2010). "Virtual organization for supply chain integration: Two cases in the textile and fashion retailing industry". International Journal of Production Economics, vol. 127.

Wareham, J.; Mahthiassen, L.; Rai, A.; Straub, D. y R. Klein (2005). "The business value of digital supply networks: a program of re- 
search on the impacts of globalization". Journal of International Management, vol. 11.

Wu, Y.; Frizelle, G. y J. Efstathiou (2007). "A study on the cost of operational complexity in customer - supplier systems". International Journal of Production Economics, vol. 106.

Yang, S. y T. Li (2002). "Agility evaluation of mass customization product manufacturing". Journal of Materials Processing Technology, vol. 129. 